\title{
Defective dual varieties for real spectra
}

\author{
Jens Forsgård ${ }^{1}$
}

Received: 9 October 2017 / Accepted: 6 February 2018 / Published online: 27 February 2018

(C) The Author(s) 2018. This article is an open access publication

\begin{abstract}
We introduce an invariant of a finite point configuration $A \subset \mathbb{R}^{1+n}$ which we denote the cuspidal form of $A$. We use this invariant to extend Esterov's characterization of dual-defective point configurations to exponential sums; the dual variety associated with $A$ has codimension at least 2 if and only if $A$ does not contain any iterated circuit.
\end{abstract}

Keywords A-Discriminant · Dual defective · Toric geometry · Fewnomials · Exponential sums

Mathematics Subject Classification Primary 14M25; Secondary 55R80 · 52B35

\section{Introduction}

The main undertaking of fewnomial theory is to bound the number of connected components of the positive part of a variety defined by a system of equations solely in terms of the number of variables $n$ and the total number of monomials $N$ appearing in the system. Since the constitutive monograph [12] of Khovanskiı̌, fewnomial theory has often been studied alongside the theory of exponential sums. After all, the coordinatewise exponential map exp: $\mathbb{R}^{n} \rightarrow \mathbb{R}_{+}^{n}$ is a diffeomorphism, and when replacing monomials $z^{\alpha}$ by exponentials $e^{\langle w, \alpha\rangle}$ the fundamental examples (read: Descartes' rule of signs) remain valid.

Fewnomial theory has also been studied using Gel'fand, Kapranov, and Zelevinsky's "A-philosophy." In this approach, one considers the family of all polynomials

\footnotetext{
$凶 \quad$ Jens Forsgård

Jens.Forsgaard@unige.ch

1 Mathématiques, Université de Genève, Villa Battelle, 1227 Carouge, Switzerland
} 
which can be expressed using a fixed set (the support set) $A$ of exponent vectors $\alpha$. This family is studied using $A$-discriminants and Gale duality. See, e.g., $[1,4,9]$.

However, an "A-philosophy" for exponential sums has not been properly developed. We are aware only of [15], where the A-discriminant and its Horn-Kapranov uniformization were generalized to the case of exponential sums. We put ourself in this setting and consider the family

$$
\left(\mathbb{C}^{\times}\right)^{A}=\left\{f(w)=\sum_{\alpha \in A} c_{\alpha} e^{\langle w, \alpha\rangle} \mid c_{\alpha} \in \mathbb{C}^{\times}\right\},
$$

where $A \subset \mathbb{R}^{1+n}$ is a finite set, and $N=\# A$. We have many names for those we love; the support set $A$ is also known as the Bohr spectrum (or, simply, spectrum) of an exponential sum $f \in\left(\mathbb{C}^{\times}\right)^{A}$.

The work presented in this article emerged from an innocent question of whether a theorem of Katz [11] holds also in the framework of exponential sums. The answer is affirmative, as we show in Theorem 3.5. This theorem has already seen an application in [7] to reduce a fewnomial hypersurface bound from exponential to subexponential in the dimension. Our main line of thought goes, however, in a slightly different direction.

We associate to the spectrum $A \subset \mathbb{R}^{1+n}$ a combinatorial invariant in the form of a homogeneous polynomial $P_{A}(t)$ of degree $n$, which we call the cuspidal form of $A$, see Definition 3.1. Here, $t$ denotes the parameters of the Horn-Kapranov uniformization of the $A$-discriminant $\check{X}_{A}$, and hence, the cuspidal form depends also on a choice of Gale dual of $A$. The name "cuspidal form" reflects the fact that $P_{A}(t)$ describes the preimage of the cuspidal locus of $\check{X}_{A}$ under the Horn-Kapranov uniformization. In particular, as one observes immediately, the configuration $A$ is dual defective if and only if $P_{A}(t)$ vanishes identically.

The core part of this work is to describe the properties of the cuspidal form $P_{A}(t)$ as an invariant of the spectrum $A$. Our main technical results are concerned with factorizations. For example, if $A$ is a diagonal configuration (Definition 4.3), then the cuspidal form $P_{A}(t)$ factors as a product of the cuspidal forms of the diagonal configurations of $A$.

We spend a fair amount of energy describing linear factors of $P_{A}(t)$. These are of two distinct types. The first type consists of linear factors corresponding to rows of the Gale dual. These factors correspond to points $\alpha \in A$ such that $A \backslash\{\alpha\}$ is dual defective. Such factors are studied in Sects. 4.1 and 5. The complementary type corresponds to discriminant varieties embedded into the cuspidal locus of $\check{X}_{A}$. This generalizes results of [13], where the cuspidal form first appeared in the special case of $n=1$ (when $P_{A}(t)$ is itself a linear form).

As an application, we extend Esterov's characterization from $[5,6]$ of dual-defective point configurations to the case of exponential sums: A point configuration $A$ is dual defective if and only if it does not contain any iterated circuit (Theorem 6.4). Our proof uses in addition to the properties of the cuspidal form only the pigeonhole principle.

Finally, in Sect. 8 we study the special case $n=2$ when $P_{A}(t)$ is a quadratic form. We consider the rank and the signature of $P_{A}(t)$, which are invariant under the choice of Gale dual. It turns out (Theorem 8.1) that the rank of $P_{A}(t)$ is at most 3, independent 
of the number of variables $m$. The degenerate case that the rank is at most 2 occurs if and only if the spectrum $A$ is contained in a conic section; the type of the conic section is described by the signature of $P_{A}(t)$. Spectra $A$ contained in conic sections have appeared in the study of self-dual toric varieties; see, e.g., the Togliatti surface in $[3, \S 4.1]$, whose spectrum consists of six points on an ellipse.

It is possible to consider, for example, complex instead of real spectra. The $A$ discriminant variety, in the sense of exponential sums, remains well defined. The characterization of dual-defective point configurations as those who do not contain an iterated circuit remains valid. However, for complex spectra, the relationship to combinatorics is lost, as we cannot, for example, talk about Newton polygons. We have chosen to stay in the real world as this is the most interesting case for applications.

\section{Prelude: Gale duality and the Horn-Kapranov uniformization}

In this section, we introduce notation and briefly describe some standard constructions and results associated with $A$-discriminants and adapt them to the setting of exponential sums.

\subsection{The setup}

Recall that $A$ is a finite subset of $\mathbb{R}^{1+n}$ with $N$ elements. By ordering the elements of $A$, we obtain an isomorphism $\mathbb{C}^{A} \simeq \mathbb{C}^{N}$. With an ordering chosen, by abuse of notation, we identify $A$ with the $(1+n) \times N$-matrix $A=\left(\alpha_{1}, \ldots, \alpha_{N}\right)$. Doing so, we often write $c_{k}$ for the coefficient of $e^{\left\langle w, \alpha_{k}\right\rangle}$ and identify an exponential sum $f$ with its (column) coefficient vector $c=\left(c_{1}, \ldots, c_{N}\right)^{\mathrm{T}}$.

The properties of the exponential sum $f$ which we are interested in (e.g., the existence of a singular point) are invariant of linear changes of variables. That is, if we denote by $M_{N, n}(\mathbb{R})$ the space of all such matrices $A$, then we are interested in the orbits under the left action of $\mathrm{GL}_{1+n}(\mathbb{R})$.

If $A \subset \mathbb{Z}^{1+n}$, then the exponential sum is said to be polynomial, and the configuration $A$ is said to be algebraic. In this case, we can associate to $f$ the $(1+n)$-variate polynomial $g(z)=\sum_{\alpha \in A} c_{\alpha} z^{\alpha}$. The analytic variety $Z(f)$ is an infinite covering of the quasi-affine variety $Z(g) \subset\left(\mathbb{C}^{\times}\right)^{1+n}$. We often descend to the algebraic case in examples.

Two natural assumptions are imposed. Firstly, $A$ is assumed to be pseudohomogeneous. That is, we assume the existence of a linear form $\xi \in \operatorname{Hom}\left(\mathbb{R}^{1+n}, \mathbb{R}\right)$ such that $\langle\xi, \alpha\rangle=1$ for all $\alpha \in A$. Secondly, it is assumed that $\xi$ is unique. These two assumptions are equivalent to that the matrix $A$ is of full rank (equal to $1+n$ ) with the all ones vector in its row span. In particular, the Newton polygon $\mathscr{N}=\operatorname{Conv}\left(\alpha_{1}, \ldots, \alpha_{N}\right)$ has dimension $n$. We do not assume that the columns of $A$ are distinct.

We associate to $A$ the map $\exp _{A}$ given by

$$
\exp _{A}: \mathbb{C}^{1+n} \rightarrow\left(\mathbb{C}^{\times}\right)^{N}, \quad w \mapsto\left(e^{\left\langle w, \alpha_{1}\right\rangle}, \ldots, e^{\left\langle w, \alpha_{N}\right\rangle}\right)^{\mathrm{T}}
$$


where $\mathbb{C}^{N}$ should be considered as the dual space of $\mathbb{C}^{A}$. In the algebraic case, the map $\exp _{A}$ parametrizes a torus. By taking Zariski closure, one obtains a toric variety denoted $X_{A}$. Its projectively dual $\check{X}_{A}$ is known as the A-discriminantal variety, see [8]. In the case that $A$ is algebraic and $\check{X}_{A}$ is a hypersurface, its defining polynomial $D_{A}$ is called the $A$-discriminant. In the algebraic case, the locus $D_{A}(c)=0$ is the closure of the locus of all $g$ which has a singular point in $\left(\mathbb{C}^{\times}\right)^{1+n}$. In the general case, we define $\check{X}_{A} \subset\left(\mathbb{C}^{\times}\right)^{A}$ by that $f \in \check{X}_{A}$ if and only if the exponential sum $f$ has a singular point in $\mathbb{C}^{1+n}$. Notice that we do not take a Zariski closure in the general case, as $\check{X}_{A} \subset\left(\mathbb{C}^{\times}\right)^{A}$ is not necessarily algebraic for real spectra.

Example 2.1 A point configuration $A$ is said to be a pyramid if all points but one is contained in some strict affine subspace. It is straightforward to check that if $A$ is a pyramid, then the discriminant locus $\check{X}_{A}$ is empty.

Example 2.2 The codimension $m$ of a spectrum $A$ is given by $m=N-n-1$. A point configuration of codimension 1 , which is not a pyramid, is said to be a circuit. Let us consider a circuit in two dimensions. Using the $\mathrm{GL}_{1+n}(\mathbb{R})$-action, we can assume that $A$ is of the form

$$
A=\left(\begin{array}{cccc}
1 & 1 & 1 & 1 \\
0 & 1 & 0 & \alpha_{11} \\
0 & 0 & 1 & \alpha_{12}
\end{array}\right)
$$

The formula of the discriminant $D_{A}(c)$ in the algebraic case, from [8, p. 274], generalizes to the binomial exponential expression

$$
D_{A}(c)=c_{3}\left(\frac{c_{0}}{\alpha_{11}+\alpha_{12}-1}\right)^{\alpha_{11}+\alpha_{12}-1}-\left(\frac{-c_{1}}{\alpha_{11}}\right)^{\alpha_{11}}\left(\frac{-c_{2}}{\alpha_{12}}\right)^{\alpha_{12}} .
$$

The multivaluedness of the exponential functions requires some caution when handling this expression. For this reason, we refrain from using the $A$-discriminant $D_{A}(c)$ in our analysis.

Remark 2.3 In examples, it is more convenient to consider a family of inhomogeneous $n$-variate exponential sums. For an exponential sum $f$ in the variables $z_{1}, \ldots, z_{n}$, the corresponding pseudo-homogeneous exponential sum is given by $e^{z_{0}} f$. For a family of $n$-variate inhomogeneous polynomials with support set $A$, this corresponds to adjoining a top row of all ones in its matrix representation. In this case, $\xi=$ $(1,0, \ldots, 0)$.

\subsection{The Horn-Kapranov uniformization}

Our main tool is the Horn-Kapranov uniformization of the A-discriminant hypersurface from [10]. For exponential sums, this map was deduced in [15, Thm. 1.7]. Step-by-step, the standard deduction of the Horn-Kapranov uniformization ( $c f$. [2, Prop. 4.1]) is sufficient to cover also the case of exponential sums.

A Gale dual of $A$ is a matrix $B$ yielding an exact sequence of $\mathbb{C}$-vector spaces 


$$
0 \longrightarrow \mathbb{C}^{m} \stackrel{B}{\longrightarrow} \mathbb{C}^{A} \stackrel{A}{\longrightarrow} \mathbb{C}^{1+n} \longrightarrow 0 .
$$

Since $A$ has full rank, the rank of $B$ is $m$. Let us denote the rows of $B$ by $\beta_{k}$ for $k=1, \ldots, N$, and let us introduce coordinates $t$ in $\mathbb{C}^{m}$. Let $\mathbb{C}_{B}^{m}$ denote the inverse image of $\left(\mathbb{C}^{\times}\right)^{A}$ under the map $B$. The exceptional locus $\mathscr{H}_{B}=\mathbb{C}^{m} \backslash \mathbb{C}_{B}^{m}$ is the union of the linear subspaces $Z\left(\beta_{k}\right)$. Assuming that $A$ is not a pyramid (i.e., assuming that no $\left.\beta_{k}=0\right)$, then $\mathscr{H}_{B}$ is a central hyperplane arrangement.

Theorem 2.4 (Kapranov, Rojas-Rusak) The dual variety $\check{X}_{A}$ is parametrized by the map

$$
\Phi:\left(\mathbb{C}^{\times}\right)^{1+n} \times \mathbb{C}_{B}^{m} \rightarrow\left(\mathbb{C}^{\times}\right)^{A}, \quad \Phi(\omega ; t)=\exp _{A}(\omega) *(B t)
$$

where $*$ denotes componentwise multiplication.

The exponential sum $f=\Phi(\omega ; t)$ has a singular point with coordinates $w=-\omega$.

The map $\Phi$ is far from injective as, for example, the parameter space and $\left(\mathbb{C}^{\times}\right)^{A}$ have the same dimensions. However, that $\Phi$ is homogeneous in $t$ and the existence of the linear form $\xi$ implies that $\Phi$ parametrizes a strict (multivalued) analytic subvariety of $\left(\mathbb{C}^{\times}\right)^{A}$.

Remark 2.5 In the algebraic case, the $A$-discriminantal polynomial has $1+n$ homogeneities, arising from the matrix $A$. In Kapranov's paper [10], these homogeneities were removed by composition with the map $\exp _{B}:\left(\mathbb{C}^{\times}\right)^{A} \rightarrow\left(\mathbb{C}^{\times}\right)^{m}$. The composite map is, in this algebraic case, a rational function of $t$. To avoid real powers of linear forms, we settle for the map $\Phi$ of Theorem 2.4.

Let us end this section with a well-known property of Gale duality. For a square matrix $M$, we denote its determinant by $|M|$.

Lemma 2.6 ([14, Lem. 2.10]) Let $B$ be any Gale dual of $A$, and let $\sigma \subset[N]$ be a choice of $1+n$ indices with sign $\operatorname{sgn}(\sigma)=\prod_{k \in \sigma}(-1)^{k}$. Denote by $A_{\sigma}$ the maximal cofactor of $A$ obtained by keeping the columns indexed by $\sigma$ and let $B_{\sigma}$ denote the complementary maximal cofactor of $B$ obtained by deleting the rows indexed by $\sigma$. Then, there is a nonzero constant $C(B)$, independent of $\sigma$, such that

$$
\left|A_{\sigma}\right|=C(B) \operatorname{sgn}(\sigma)\left|B_{\sigma}\right|
$$

Remark 2.7 In the algebraic case, it is natural to assume that $\mathbb{Z} A=\mathbb{Z}^{1+n}$, in which case $\bar{A}$ can be chosen as an integer unimodular matrix. That is, also $\bar{B}$ is integer unimodular, and hence, $B$ is a Gale dual in the combinatorial sense. In this case, we have that $C(B)= \pm 1$, depending on $B$.

\section{The cuspidal form}

In this section, we define the cuspidal form $P_{A}(t)$. To simplify notation, we impose the assumption that the top row of $A$ is equal to the all ones vector, and we let $\hat{A}$ denote the $n \times N$ matrix obtained from $A$ by deleting the top row. 
Let $B$ denote a Gale dual of $A$. In slight deviation from the notation in Lemma 2.6, we let $\Sigma$ denote the set of all subsets $\sigma \subset[N]$ of cardinality $n$. For each $\sigma \in \Sigma$, let $\hat{A}_{\sigma}$ denote the maximal cofactor of $\hat{A}$ obtained by deleting all columns corresponding to indices $k \notin \sigma$.

Definition 3.1 We define the cuspidal form $P_{A}(t)$ to be

$$
P_{A}(t)=\sum_{\sigma \in \Sigma}\left|\hat{A}_{\sigma}\right|^{2} \prod_{k \in \sigma}\left\langle\beta_{k}, t\right\rangle
$$

The vanishing locus of $P_{A}(t)$ will be called the cuspidal locus in $\mathbb{C}^{m}$. We say that the $P_{A}(t)$ is trivial if it vanishes identically.

The cuspidal form appeared in unpublished works of Dickenstein, Feichtner, and Sturmfels (Dickenstein, personal communication, 2017) in the special case $n=1$, it was considered in [13].

We have that $P_{A}(t)$ is a homogeneous form of degree $n$ in the coordinates $t$. Hence it defines, in the case that it is nontrivial, a hypersurface in $\mathbb{P}^{m-1}$. (Except, of course, for the case $m=1$.)

The cuspidal form $P_{A}(t)$ depends on the choice of Gale dual $B$. As two Gale duals differ by a change of coordinates in $\mathbb{C}^{m}$, we consider this dependence to be implicitly understood from that $P_{A}(t)$ is written as a polynomial in the variables $t$. Since we can choose coordinates in $\mathbb{C}^{m}$ arbitrarily, one cannot, in general, ask for a combinatorial interpretation of the coefficients of $P_{A}(t)$. When there is a canonical choice of coordinates, however, there are reasonable interpretations.

Example 3.2 Let $A$ be a pyramid. That is, there is a point $\alpha \in A$ such that $A \backslash\{\alpha\}$ is contained in an affine space of dimension $n-1$. This is equivalent to that $\beta_{\alpha}=0$. Thus, if $\alpha \in \sigma$, then $\left\langle\beta_{\alpha}, t\right\rangle=0$, and if $\alpha \neq \sigma$ then $\left|\hat{A}_{\sigma}\right|=0$. Hence, each term of (1) vanishes, implying that $P_{A}(t)$ vanishes identically.

Example 3.3 Let $m=1$, and let $A_{k}$ denote the $(n+1) \times(n+1)$ submatrix of $A$ obtained by deleting the $k$ th entry and assume, without loss of generality, that $\alpha_{1}=(1,0, \ldots, 0)^{\mathrm{T}}$. Then, each $\sigma \in \Sigma$ containing 1 has $\left|\hat{A}_{\sigma}\right|=0$, and each $\sigma$ not containing 1 has $\left|\hat{A}_{\sigma}\right|=\left|A_{k}\right|$ where $k=k(\sigma)$ is the unique index greater than 1 not contained in $\sigma$. By Lemma 2.6, we can choose a Gale dual $B$ according to the rule that $\beta_{k}=(-1)^{k}\left|A_{k}\right|$. Hence,

$$
P_{A}(t)=t^{n}\left(\prod_{k>1}(-1)^{k}\left|A_{k}\right|\right) \sum_{k>1}(-1)^{k}\left|A_{k}\right|=\operatorname{sgn}(n) t^{n} \prod_{k=0}^{n+2}\left|A_{k}\right|
$$

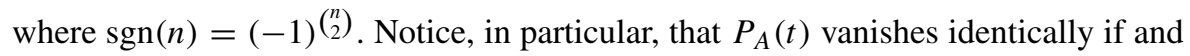
only if $\left|A_{k}\right|=0$ for some $k$, which is equivalent to that $A$ is a pyramid. 


\subsection{The cuspidal locus of $\check{X}$}

Recall the assumption that the top row of $A$ consists of the all ones vector. Let us dehomogenize $\Phi$ by setting $\omega_{0}=1$. That is, we consider the parametrization map $\Phi:\left(\mathbb{C}^{\times}\right)^{n} \times \mathbb{C}_{B}^{m} \rightarrow \check{X}_{A}$. In standard terminology, a point $f=\Phi(\omega ; t) \in \check{X}_{A}$ is a cusp of $\check{X}_{A}$ if and only if the image of the pushforward

$$
\mathrm{d} \Phi: T_{(w ; t)}\left(\left(\mathbb{C}^{\times}\right)^{n} \times \mathbb{C}_{B}^{m}\right) \rightarrow T_{f} \check{X}_{A}
$$

is an affine space of dimension at most $N-2=n+m-1$. That is, $f$ is a cusp if and only if the Jacobian matrix

$$
J_{\Phi}(\omega ; t)=\left(\begin{array}{ccc}
\alpha_{11}\left\langle\beta_{1}, t\right\rangle e^{\left\langle\omega, \alpha_{1}\right\rangle} & \ldots & \alpha_{N 1}\left\langle\beta_{N}, t\right\rangle e^{\left\langle\omega, \alpha_{N}\right\rangle} \\
\vdots & \ddots & \vdots \\
\alpha_{1 n}\left\langle\beta_{1}, t\right\rangle e^{\left\langle\omega, \alpha_{1}\right\rangle} & \ldots & \alpha_{N n}\left\langle\beta_{N}, t\right\rangle e^{\left\langle\omega, \alpha_{N}\right\rangle} \\
\beta_{11} e^{\left\langle\omega, \alpha_{1}\right\rangle} & \ldots & \beta_{N 1} e^{\left\langle\omega, \alpha_{N}\right\rangle} \\
\vdots & \ddots & \vdots \\
\beta_{1 m} e^{\left\langle\omega, \alpha_{1}\right\rangle} & \ldots & \beta_{N m} e^{\left\langle\omega, \alpha_{N}\right\rangle}
\end{array}\right) .
$$

has rank at most $n+m-1$. (We drop the subindex $\Phi$ in the notation.)

Theorem 3.4 We have that $f$ is a cusp of $\check{X}_{A}$ if and only if there exists a $(w, t) \in$ $\left(\mathbb{C}^{\times}\right)^{n} \times \mathbb{C}_{B}^{m}$ such that $f=\Phi(\omega ; t)$ and $P_{A}(t)=0$.

Proof We need to determine when the maximal minors of $J(\omega ; t)$ vanish. This does not depend on $\omega$ : the common factors $e^{\left\langle\omega, \alpha_{k}\right\rangle}$ of each column of $J(\omega ; t)$ can be factored outside of any minor. We compute the maximal minors of $J(1 ; t)$.

For each submatrix $S \subset A$ obtained by deleting any number of columns, let $\hat{S}$ denote the matrix $S$ with the top row (consisting of all ones) deleted. In particular, $\hat{\alpha}$ denotes the column of $\hat{A}$ corresponding to the column $\alpha \in A$.

Let $J_{k}$ denote the maximal minor of $J(1 ; t)$ obtained by deleting the $k$ th column. Without loss of generality, we impose two assumptions. Firstly, as permuting the columns only alters the value of a minor by \pm 1 , we can assume that $k=1$. Secondly, we assume that $\alpha_{11}=\cdots=\alpha_{1 n}=0$ (i.e., that $\left.\alpha_{1}=(1,0 \ldots, 0)^{\mathrm{T}}\right)$. Indeed, multiplying $J(1 ; t)$ from the left by

$$
T=\left(\begin{array}{cc}
I_{n} & -\hat{\alpha}_{1} t^{\mathrm{T}} \\
0 & I_{m}
\end{array}\right)
$$

corresponds to the translation $\hat{\alpha} \mapsto \hat{\alpha}-\hat{\alpha}_{1}$. As $|T|=1$, all maximal minors are invariant under this action.

Let $\Sigma^{\prime}$ denote the set of all subsets $\sigma \subset[N] \backslash\{1\}$ of cardinality $n$, and for each $\sigma \in \Sigma^{\prime}$ set $\bar{\sigma}=\{1\} \cup \sigma$. By the Laplace expansion in complementary minors (of sizes $n \times n$ and $m \times m$ ) 


$$
J_{1}=\sum_{\sigma \in \Sigma^{\prime}} \operatorname{sgn}(\sigma)\left(\prod_{k \in \sigma}\left\langle\beta_{k}, t\right\rangle\right)\left|\hat{A}_{\sigma}\right|\left|B_{\bar{\sigma}}\right| .
$$

We make three observations. Firstly, since $\alpha_{1}=(1,0, \ldots, 0)^{\mathrm{T}}$, we have that $\left|A_{\bar{\sigma}}\right|=$ $\left|\hat{A}_{\sigma}\right|$. Secondly, according to Lemma 2.6 (assuming that $C(B)=1$ ), we have that $\left|B_{\bar{\sigma}}\right|=\operatorname{sgn}(\bar{\sigma})\left|A_{\bar{\sigma}}\right|$. Thirdly, we have that $\operatorname{sgn}(\sigma)=\operatorname{sgn}(\bar{\sigma})$. All in all, we conclude that

$$
J_{1}=\sum_{\sigma \in \Sigma^{\prime}}\left|\hat{A}_{\sigma}\right|^{2}\left(\prod_{k \in \sigma}\left\langle\beta_{k}, t\right\rangle\right) .
$$

Since $\hat{\alpha}_{1}=0$, we have that $\left|\hat{A}_{\sigma}\right|=0$ for any $\sigma$ with $1 \in \sigma$. Hence, $J_{1}=P_{A}(t)$.

\subsection{Katz' theorem}

In the algebraic case, it follows from Katz' dimension formula [11], see also [8, §1.5], that the Jacobian $J(\omega ; t)$ has full rank at $(\omega ; t)$ if and only if the Hessian matrix $H_{f}(w)$ of $f=\Phi(\omega ; t)$ at the point $w=-\omega$ is singular. This statement remains true if one generalizes to exponential sums. It is possible to obtain a proof of this fact by making the appropriate modifications to the exposition in $[8, \S 1.5]$; since we have introduced the cuspidal form (1), we prefer the following more direct approach.

Theorem 3.5 Let $f=\Phi(\omega ; t) \in \check{X}_{A}$. Then, the Hessian matrix $H_{f}(w)$ is singular at $w=-\omega$ if and only if $P_{A}(t)=0$. In particular, if $\check{X}_{A}$ is a hypersurface and $f$ is a smooth point on $\check{X}_{A}$, then the Hessian matrix $H_{f}(-\omega)$ is nonsingular.

Proof We only need to prove the first part. As in the proof of Theorem 3.4, the torus action can be quoted out. Hence, it suffices to consider the case $\omega=0$, where the singular point is located at $w=0$. We have that

$$
f_{k l}^{\prime \prime}(0)=\sum_{j=1}^{N} \alpha_{j k} \alpha_{j l}\left\langle\beta_{j}, t\right\rangle, \quad 1 \leq k, l \leq n .
$$

In particular, the $p$ th column $H_{p}$ of $H_{f}(1)$, for $p=1, \ldots, n$ is a sum of $N$ vectors

$$
H_{p}=\sum_{j=1}^{N} \alpha_{j p}\left\langle\beta_{j}, t\right\rangle \hat{\alpha}_{j}
$$

For any two such columns, the $j$ th summands are both multiples of $\hat{\alpha}_{j}$. In particular, we can expand the determinant

$$
\left|H_{f}(0)\right|=\sum_{\sigma \in \Sigma} \sum_{\pi}\left|\alpha_{k \pi(k)}\left\langle\beta_{k}, t\right\rangle \hat{\alpha}_{k}\right|_{k \in \sigma}
$$


where $\pi$ runs over the set of all bijections $\pi: \sigma \rightarrow[n]$. It follows that

$$
\left|H_{f}(0)\right|=\sum_{\sigma \in \Sigma}\left(\prod_{k \in \sigma}\left\langle\beta_{k}, t\right\rangle\right) \sum_{\pi}\left|\alpha_{k \pi(k)} \hat{\alpha}_{k}\right|_{k \in \sigma}=\sum_{\sigma \in \Sigma}\left(\prod_{k \in \sigma}\left\langle\beta_{k}, t\right\rangle\right)\left|\hat{A}_{\sigma}\right|^{2}
$$

which completes the proof.

\section{Properties of the cuspidal form}

In this section, we investigate the cuspidal form $P_{A}(t)$ as a function of the spectrum A.

\subsection{Subconfigurations of $A$}

We denote by $Z(\beta)$ the hyperplane in $\mathbb{C}^{m}$ with equation $\langle\beta, t\rangle=0$.

Theorem 4.1 Let $\alpha \in A$ be such that $A \backslash\{\alpha\}$ has rank $1+n$, let $t^{\prime}$ be coordinates in $Z(\beta)$ and $t=\left(t^{\prime}, t_{m}\right)$ coordinates in $\mathbb{C}^{m}$. Then,

$$
P_{A \backslash\{\alpha\}}\left(t^{\prime}\right)=\left.P_{A}(t)\right|_{Z(\beta)} .
$$

Proof By assumption, if we write $A=\left(A^{\prime}, \alpha\right)$, then $A^{\prime}$ is of rank $1+n$ and codimension $m-1$. We can chose a Gale dual $B$ of $A$ of the form

$$
B=\left(\begin{array}{cc}
B^{\prime} & * \\
0 & 1
\end{array}\right)
$$

where $B^{\prime}$ is a Gale dual of $A^{\prime}$. The theorem follows from that the restriction of $P_{A}(t)$ to $Z(\beta)$ is given by $t_{m}=0$. First, for any $\sigma \in \Sigma$ with $N \in \sigma$, we have that the term of $P_{A}(t)$ corresponding to $\sigma$ has the monomial $t_{m}$ as a factor. Second, we have that $\left\langle\beta_{k}, t\right\rangle$ restricted to $t_{m}=0$ equals $\left\langle\beta_{k}^{\prime}, t^{\prime}\right\rangle$ for any $k=1, \ldots, N-1$.

Corollary 4.2 If $A^{\prime} \subset A$ is such that $P_{A^{\prime}}$ is nontrivial, then $P_{A}$ is nontrivial.

\subsection{Diagonal and upper triangular configurations}

Definition 4.3 We say that a point configuration $A$ is upper triangular if it can, after acting by $\mathrm{GL}_{1+n}(\mathbb{R})$, be written in the form

$$
A=\left(\begin{array}{ccccc}
1 & 1 & 1 & \cdots & 1 \\
0 & \tilde{A}_{1} & * & \cdots & * \\
0 & 0 & \tilde{A}_{2} & \cdots & * \\
\vdots & \vdots & \vdots & \ddots & \vdots \\
0 & 0 & 0 & \cdots & \tilde{A}_{m}
\end{array}\right)
$$


where we associate to each $\tilde{A}_{j}$ the point configuration $A_{j}$ defined by $\hat{A}_{j}=\left(0, \tilde{A}_{j}\right)$. The configurations $A_{j}$ for $j=1, \ldots m$ are called the diagonal configurations of $A$. If all elements marked by $*$ vanish, then the point configuration is said to be diagonal.

Remark 4.4 A configuration $A$ is an iterated circuit in the sense of Esterov [5, Def. 3.15] if and only if after applying an integer affine transformation it is an upper triangular configuration all of whose diagonal configurations are circuits.

Theorem 4.5 Let $A$ be a diagonal point configuration with diagonal configurations $A_{1}, \ldots, A_{m}$. Then, $P_{A}(t)=P_{A_{1}}\left(t_{1}\right) \cdots P_{A_{m}}\left(t_{m}\right)$.

Proof By induction, it suffices to consider the case $m=2$. For a minor $\left|\hat{A}_{\sigma}\right|$ of $\hat{A}$ to be nonvanishing, it must hold that the set $\sigma \in \Sigma$ consists of $n_{j}$ columns corresponding to points in $\tilde{A}_{j}$ for $j=1,2$. Therefore, for each nonvanishing term of $P_{A}(t)$ we have the factorization

$$
\left|A_{\sigma}\right|=\left|A_{\sigma_{1}}\right|\left|A_{\sigma_{2}}\right|
$$

where $\sigma_{j} \in \Sigma_{j}$ for $j=1,2$ and $\sigma=\sigma_{1} \cup \sigma_{2}$. We can write $A_{j}$ and its Gale dual in the block forms

$$
A_{j}=\left(\begin{array}{cc}
1 & 1 \\
0 & \tilde{A}_{j}
\end{array}\right) \quad \text { and } \quad B_{j}=\left(\begin{array}{c}
* \\
\tilde{B}_{j}
\end{array}\right) .
$$

Then, a Gale dual of $A$ can be written as

$$
B=\left(\begin{array}{cc}
* & * \\
\tilde{B}_{1} & 0 \\
0 & \tilde{B}_{2}
\end{array}\right)
$$

Hence, for each $\sigma=\sigma_{1} \cup \sigma_{2}$ it holds that

$$
\prod_{k \in \sigma}\left\langle\beta_{k}, t\right\rangle=\left(\prod_{k \in \sigma_{1}}\left\langle\beta_{k}, t_{1}\right\rangle\right)\left(\prod_{k \in \sigma_{2}}\left\langle\beta_{k}, t_{2}\right\rangle\right),
$$

where we, by abuse of notation, interpret $\beta_{k}$ as a row both of $B$ and of $\tilde{B}_{j}$ when $k \in \sigma_{j}$.

Remark 4.6 Let $A$ be an iterated circuit. By Example 3.3, we have that $P_{A_{j}}\left(t_{j}\right)$ is a nontrivial monomial in the single variable $t_{j}$ of degree $n_{j}$ for each $j=1, \ldots, m$. It is straightforward to check that the coefficient of the monomial $t_{1}^{n_{1}} t_{2}^{n_{2}} \cdots t_{m}^{n_{m}}$ is unchanged if one deletes all the elements of $A$ marked by a star in (2). In particular, it follows from Theorem 4.5 that the monomial of $P_{A}(t)$ with exponent vector $\left(n_{1}, n_{2}, \ldots, n_{m}\right)$ is given by $P_{A_{1}}\left(t_{1}\right) \cdot P_{A_{2}}\left(t_{2}\right) \cdots P_{A_{m}}\left(t_{m}\right)$. In particular, $P_{A}(t)$ is nontrivial in this case. 


\section{Linear factors}

Corollary 5.1 Let $\alpha \in A$. If the cuspidalform $P_{A \backslash\{\alpha\}}$ vanishes identically, then $\left\langle\beta_{\alpha}, t\right\rangle$ divides $P_{A}(t)$.

Proof This follows from Theorem 4.1 and Hilbert's Nullstellensatz.

Example 5.2 Consider the point configuration and Gale dual

$$
A=\left(\begin{array}{lllll}
1 & 1 & 1 & 1 & 1 \\
0 & 1 & 2 & 0 & 1 \\
0 & 0 & 0 & 1 & 2
\end{array}\right) \quad \text { and } \quad B^{\mathrm{T}}=\left(\begin{array}{rrrrr}
2 & -1 & 0 & -2 & 1 \\
1 & -2 & 1 & 0 & 0
\end{array}\right)
$$

Deleting any of the two last columns of $A$, we obtain a pyramid, which has trivial cuspidal form by Example 3.2. Notice that the corresponding rows of the Gale dual $B$ are parallel. The cuspidal form in this case is $P_{A}(t)=4 t_{1}\left(t_{2}-t_{1}\right)$. In particular, Corollary 5.1 cannot be extended to a bijective correspondence between $\alpha \in A$ with $P_{A \backslash\{\alpha\}}$ trivial and linear factors of $P_{A}(t)$.

Proposition 5.3 Let $A$ be a point configuration such that $P_{A}(t)$ is nontrivial. Assume that one (and therefore every) Gale dual $B$ has a set of $k$ parallel rows $\beta_{1}, \ldots, \beta_{k}$. Let $\beta_{j}=\gamma_{j} \beta$ for some $\beta \neq 0$ and scalars $\gamma_{j}$ for $j=1, \ldots, k$. Then, $\langle\beta, t\rangle^{k-1}$ divides $P_{A}(t)$. If, in addition, $\beta_{1}+\cdots+\beta_{k}=0$, then $\langle\beta, t\rangle^{k}$ divides $P_{A}(t)$.

Proof Let the columns $\alpha_{1}, \ldots, \alpha_{k}$ of $A$ correspond to the $k$ rows $\beta_{1}, \ldots, \beta_{k}$ of $B$. By Lemma 2.6, any maximal minor of $A$ not containing all but at most one of the columns $\alpha_{1}, \ldots, \alpha_{k}$ vanishes. In particular, the remaining points $\hat{\alpha}_{k+1}, \ldots, \hat{\alpha}_{N}$ are contained in some $n-k+1$-dimensional affine subspace. Since $A$ has full rank, we can write $A$ in the form (where we have reordered so that $\alpha_{1}, \ldots, \alpha_{k}$ are the $k$ last columns)

$$
A=\left(\begin{array}{ccc}
1 & 1 & 1 \\
\hat{A}^{\prime} & * & * \\
0 & I_{k-1} & \gamma
\end{array}\right) \text { and } B=\left(\begin{array}{cc}
B^{\prime} & * \\
0 & -\gamma \\
0 & 1
\end{array}\right)
$$

Here, $A^{\prime}$ is an $(n-k+2) \times(N-k)$-matrix with Gale dual $B^{\prime}$, and $\gamma \in \mathbb{R}^{k-1}$. Set $|\gamma|=\gamma_{1}+\cdots+\gamma_{k-1}$. We have that $\gamma_{j} \neq 0$ for each $j$, as $A$ is not a pyramid. Under these assumptions $\beta=(0, \ldots, 0,1)$, so that $\langle\beta, t\rangle=t_{m}$.

To prove the first part of the proposition, we note that any maximal minor of $\hat{A}$ which does not contain at least $k-1$ of the rightmost $k$ columns vanishes. Hence, each nonvanishing term of (1) is divisible by $t_{m}^{k-1}$.

Let us now compute the coefficient of $t_{m}^{k-1}$. If we pick a maximal minor of $\hat{A}$ containing all of the $k$ last columns, then the corresponding monomial is divisible by $t_{m}^{k}$. Hence, we need only to consider the maximal minors of $\hat{A}$ containing exactly $k-1$ of the last $k$ columns. The determinant of a $(k-1) \times(k-1)$ submatrix of $\left(I_{k-1}, \gamma\right)$ obtained by deleting the $j$ th column is, up to sign, equal to $\gamma_{j}$ if $j=1, \ldots, k-1$, and it is equal to 1 if $j=k$. Notice, also, that these $k-1$ columns give a factor $t_{m}^{k-1}$ of the corresponding term of $P_{A}(t)$. Hence, when computing the coefficient of $t_{m}^{k-1}$ 
in $P_{A}(t)$, one should, for the remaining $n-k+1$ columns of the maximal minor in question, replace the factor $\left\langle\beta_{\alpha}, t\right\rangle$ with $\left\langle\beta_{\alpha}^{\prime}, t^{\prime}\right\rangle$ where $t^{\prime}=\left(t_{1}, \ldots, t_{m-1}\right)$ and $\beta_{\alpha}^{\prime}$ is the corresponding row of $B^{\prime}$. Let $\Sigma^{\prime}$ denote the set of all subsets of $[N-k]$ of size $n-k+1$. All in all, we find that the coefficient of $t_{m}^{k-1}$ in the cuspidal form $P_{A}(t)$ is

$$
\begin{aligned}
& \sum_{\sigma \in \Sigma^{\prime}}\left|\hat{A}_{\sigma}^{\prime}\right|^{2}\left(\prod_{j \in \sigma}\left\langle\beta_{j}^{\prime}, t^{\prime}\right\rangle\right)\left(\left(\prod_{j=1}^{k-1}\left(-\gamma_{j}\right)\right)+\sum_{i=1}^{k-1} \gamma_{i}^{2} \prod_{j \neq i}\left(-\gamma_{j}\right)\right) \\
& =(-1)^{k-1} \gamma_{1} \cdots \gamma_{k-1}(1-|\gamma|) P_{\hat{A}}\left(t^{\prime}\right) .
\end{aligned}
$$

Thus, if $|\gamma|=1$, which is equivalent to the original assumption that $\beta_{1}+\cdots+\beta_{k}=0$, then $P_{A}(t)$ is divisible by $t_{m}^{k}$.

Example 5.4 Let us stay in the situation considered in Proposition 5.3. The coefficient of $t_{m}^{k-1}$ obtained at the end of that proof is

$$
(-1)^{k-1} \gamma_{1} \cdots \gamma_{k-1}(1-|\gamma|) P_{\hat{A}}\left(t^{\prime}\right),
$$

where $\gamma_{j} \neq 0$ for $j=1, \ldots, k-1$. It can happen that this coefficient vanishes even if $|\gamma| \neq 1$, as $P_{\hat{A}}\left(t^{\prime}\right)$ can be trivial. For example, consider the point configuration and Gale dual

$$
A=\left(\begin{array}{lllllllll}
1 & 1 & 1 & 1 & 1 & 1 & 1 & 1 & 1 \\
0 & 1 & 0 & 0 & 1 & 1 & 0 & 0 & 1 \\
0 & 0 & 1 & 0 & 1 & 0 & 0 & 0 & 1 \\
0 & 0 & 0 & 1 & 0 & 1 & 0 & 0 & 1 \\
0 & 0 & 0 & 0 & 0 & 0 & 1 & 0 & 3 \\
0 & 0 & 0 & 0 & 0 & 0 & 0 & 1 & 2
\end{array}\right) \text { and } B=\left(\begin{array}{rrrr}
1 & 1 & 7 \\
-1 & -1 & -1 \\
-1 & 0 & -1 \\
0 & -1 & -1 \\
1 & 0 & 0 \\
0 & 1 & 0 \\
0 & 0 & -3 \\
0 & 0 & -2 \\
0 & 0 & 1
\end{array}\right) .
$$

Here, that last three rows of the Gale dual are parallel, but they do not sum to zero. The cuspidal form corresponding to this choice of Gale dual is

$$
P_{A}(t)=6 t_{3}^{3}\left(7 t_{3}^{2}+2 t_{2} t_{3}-5 t_{2}^{2}+2 t_{1} t_{3}+2 t_{1} t_{2}-5 t_{1}^{2}\right)
$$

\section{Defect duals}

A point configuration $A$ and, in the algebraic case, the toric variety $X_{A}$, are said to be dual defective if the dual variety $\check{X}_{A}$ has codimension at least 2 . The following theorem is an immediate consequence of the properties of the Jacobian matrix.

Theorem 6.1 The point configuration A is dual defective if and only if $P_{A}(t)$ is trivial. 
Proof If $A$ is dual defective, then the rank of the Jacobian matrix $J(w ; t)$ is at most $n+m-1$ for generic $(w ; t)$. It follows that its maximal minors, which are polynomial in $(w ; t)$, vanish for generic $(w ; t)$, and hence, they vanish identically. Conversely, if $\check{X}_{A}$ is a hypersurface, as the smooth locus of $\check{X}_{A}$ is nonempty, we can find a point $f=\Phi(w ; t)$ for which the Jacobian matrix $J(w ; t)$ has rank $n+m$.

Example 6.2 Let $A$ be a pyramid. We saw in Example 3.2 that the polynomial $P_{A}(t)$ is trivial, implying (the well-known result) that $X_{A}$ is dual defective.

Proposition 6.3 If $A$ has a subset $A^{\prime}$, of full dimension, such that $A^{\prime}$ is not dual defective, then $A$ is not dual defective.

Proof This (well-known result) follows from Corollary 4.2.

Let us now recover Esterov's results on dual-defective point configuration from [5, Lem. 3.17] and [6, Cor. 3.20] and extend it from the algebraic case to the case of exponential sums.

Theorem 6.4 A spectrum $A \subset \mathbb{R}^{1+n}$ is dual defective if and only if A does not contain any iterated circuit.

Proof By Theorem 6.1 and Proposition 6.3, we translate to the equivalent statement that $P_{A}(t)$ is trivial if and only if $A$ does not contain an iterated circuit. The only if-direction is proven in Remark 4.6.

Let us prove the if-direction. Assume that $P_{A}(t)$ is nontrivial. We use a double induction over the codimension $m$ and the dimension $n$, where the base cases $m=1$ for arbitrary dimension are covered by Example 3.3.

If $P_{A \backslash\{\alpha\}}(t)$ is nontrivial for some $\alpha \in A$, then by induction on codimension, $A \backslash\{\alpha\}$ contains an iterated circuit, implying that $A$ contains an iterated circuit as well. Hence, it suffices to consider the case when $P_{A \backslash\{\alpha\}}$ is trivial for all $\alpha \in A$.

By Corollary 5.1, we have that $\langle\beta, t\rangle$ divides $P_{A}(t)$ for all rows $\beta$ of $B$. However, $B$ has $N$ rows, while $P_{A}(t)$ is a homogeneous, nontrivial polynomial of degree $n$. By the pigeonhole principle, the Gale dual $B$ must have parallel rows. For a family $\beta_{1}, \ldots, \beta_{k}$ of parallel rows of $B$, where $k>1$, let us borrow the notation from the proof of Proposition 5.3: that $\beta_{j}=\gamma_{j} \beta$ for some scalars $\gamma_{j} \neq 0$ for $j=1, \ldots, k$, and for some $\beta \neq 0$. Then, again by a comparison of the number of rows of $B$ and the degree of $P_{A}(t)$, there must exist a family, $\beta_{1}, \ldots, \beta_{k}$ of parallel rows of $B$, where $k>1$, such that $\langle\beta, t\rangle^{k}$ does not divide $P_{A}(t)$. Let us fix such a family.

Let us write $A$ and $B$ as in (3), and let $\gamma=\left(\gamma_{1}, \ldots, \gamma_{k}\right)^{\mathrm{T}}$ be as in the proof of Proposition 5.3. Since $\langle\beta, t\rangle^{k}$ does not divide $P_{A}(t)$, we have that $\left|\gamma_{k}\right| \neq 1$. Therefore, the $k \times k$-submatrix of $A$

$$
\left(\begin{array}{cc}
1 & 1 \\
I_{k-1} & \gamma
\end{array}\right)
$$

has rank $k$. (Recall that each $\gamma_{k} \neq 0$, for otherwise $A$, is a pyramid.) This has two consequences. First, we have that $\left(0, I_{k-1}, \gamma\right)$ is a $k$-dimensional circuit. Second, by applying an integer affine transformation, we can eliminate all entries marked by $*$ in 
(3). Thus, by Theorem 4.5, and with the notation of (3), it holds that $P_{A^{\prime}}(t)$ is a factor of $P_{A}(t)$. In particular, $P_{A^{\prime}}(t)$ is nontrivial, and hence, $A^{\prime}$ contains an iterated circuit of dimension $k-1$, by induction on codimension and dimension. It follows that $A$ contains an iterated circuit.

\section{Rationality of the cuspidal locus}

Let $A$ be algebraic and choose a Gale dual $B$ of $A$. We focus in this section on the reduced $A$-discriminant, whose Horn-Kapranov uniformization $\Psi_{A}(t)$ is given by $t \mapsto \Phi(1 ; t)^{B}=(B t)^{B}$, see [10] and Remark 2.5. We denote the reduced $A$ discriminant by $\check{X}_{B}$.

The case $n=1$ was studied in detail in [13], where the cuspidal form $P_{A}(t)$ appeared in this special case. For $n=1$, the cuspidal form is, for every $m$, a nontrivial linear form in $t$ vanishing along some hyperplane in $\mathbb{P}^{m-1}$. In particular, the cuspidal locus of $\check{X}_{A}$ is always unirational, and if it is a subvariety of $\check{X}_{A}$ of codimension one, then it is rational. Actually, a stronger statement holds: there is a point configuration $E$, with Gale dual $F$, such that the cuspidal locus of the reduced discriminant $X_{B}$ is isomorphic to the reduced discriminantal variety $\check{X}_{F}$. Let us here explain the corresponding result for general $n$.

Theorem 7.1 Let A be algebraic with Gale dual B. For each rational linear subspace L of $P_{A}(t)$ not contained in the exceptional locus $\mathscr{H}_{B}$ (see Sect. 2.2), there is an algebraic point configuration $E$, with Gale dual $F$, and a canonical morphism which embeds the reduced discriminant $\check{X}_{F}$ into the cuspidal locus of $\check{X}_{B}$.

Proof Express $L$ in implicit form as the image an injective linear transformation $M: \mathbb{P}^{k-1} \rightarrow \mathbb{P}^{m-1}$, where by assumption $M$ can be chosen as an integer matrix. Since $A B M=0$, we can extend $A$ (by adding rows) to an integer matrix $E$ yielding an exact sequence

$$
0 \longrightarrow \mathbb{C}^{k} \stackrel{B M}{\longrightarrow} \mathbb{C}^{A} \stackrel{E}{\longrightarrow} \mathbb{C}^{1+n+m-k} \longrightarrow 0 .
$$

It follows that $\left(\Psi_{A} \circ M\right)^{M}=\Psi_{E}$, and, hence, the morphism $\Psi_{A} \circ M \circ \Psi_{E}^{-1}$ embeds $\check{X}_{F}$ into the cuspidal locus of $\check{X}_{E}$.

The singular locus of $\check{X}_{A}$ is in general not rational. Let us give a simple example.

Example 7.2 Let $n=3$ and $N=7$, so that $P_{A}(t)$ is a cubic form in three variables. In particular, if $P_{A}(t)$ is nonsingular, then the cuspidal locus of $\check{X}_{A}$ is birational to a smooth cubic curve. Such a curve is unirational but not rational. For an explicit example when $P_{A}(t)$ is nonsingular, we present the point configuration

$$
A=\left(\begin{array}{lllllll}
1 & 1 & 1 & 1 & 1 & 1 & 1 \\
0 & 1 & 1 & 2 & 3 & 3 & 3 \\
3 & 0 & 2 & 2 & 1 & 2 & 3 \\
0 & 3 & 2 & 2 & 2 & 3 & 0
\end{array}\right)
$$




\section{The bivariate case: the signature of the quadratic form}

In the case that $n=2$ the polynomial $P_{A}(t)$ is a quadratic form. Being defined only up to choice of coordinates, let us in this section consider the (real) invariants given by the rank and the signature of $P_{A}(t)$. Recall from the literature that each real $m$-variate quadratic form is defined by a real symmetric $m \times m$-matrix $Q$. We write the signature $s_{A}$ of $P_{A}(t)$ as the triple $s_{A}=\left(s_{+}, s_{-} ; s_{0}\right)$ where $s_{ \pm}$denotes the number of positive, respectively, and negative eigenvalues of $Q$, and $s_{0}$ denotes the algebraic multiplicity of zero as an eigenvalue of $Q$. It holds that $s_{+}+s_{-}+s_{0}=m$, and the rank of $P_{A}(t)$ is given by $s_{+}+s_{-}$.

Theorem 8.1 Let $n=2$ and let $s_{A}$ denote the signature of $P_{A}(t)$. In each of the below cases, it is assumed that A does not belong to any previous cases. Then,

(i) $s_{A}=(0,0 ; m)$ if and only if $A$ is a pyramid.

(ii) $s_{A}=(1,0 ; 0)$ if and only if $A$ is contained in a nonreal parabola.

(iii) $s_{A}=(0,1 ; m-1)$ if and only if $A$ is contained in a real parabola or in two parallel lines.

(iv) $s_{A}=(1,1 ; m-2)$ if and only if $A$ is contained in a hyperbola or in two nonparallel lines.

(v) $s_{A}=(0,2 ; m-2)$ if and only if $A$ is contained in an ellipse.

(vi) $s_{A}=(1,2 ; m-3)$ in all other cases.

Remark 8.2 In the case (ii) of Theorem 8.1, it holds that $m=1$ so that $A$ consists of four points in the plane. As $P_{A}(t)$ is nontrivial, $A$ is a circuit. We can read of the signature of the circuit $A$ from Example 3.3: the Newton polygon $\mathscr{N}$ is a simplex.

More generally, as soon as $A$ has codimension $m=1$, then one can read of the combinatorial type of $A$ from the cuspidal form $P_{A}(t)$ using Example 3.3. Indeed, the Newton polygon $\mathscr{N}$ is a simplex if and only if $s_{A}=(1,0 ; 0)$, it is a quadrilateral if and only if $s_{A}=(0,1 ; 0)$, and $A$ is a pyramid if and only if $s_{A}=(0,0 ; 1)$.

To prove Theorem 8.1, we write $A$ in the form

$$
A=\left(\begin{array}{cccccc}
1 & 1 & 1 & 1 & \cdots & 1 \\
0 & 1 & 0 & \alpha_{11} & \cdots & \alpha_{m 1} \\
0 & 0 & 1 & \alpha_{12} & \cdots & \alpha_{m 2}
\end{array}\right)
$$

We write $\alpha_{k}=\left(\alpha_{k 1}, \alpha_{k 2}\right)^{\mathrm{T}}$, for simpler notation. We set $\left|\alpha_{k}\right|=\alpha_{k 1}+\alpha_{k 2}$ for all $k$. We choose the dual matrix

$$
B^{\mathrm{T}}=\left(\begin{array}{ccccccc}
\left|\alpha_{1}\right|-1 & -\alpha_{11} & -\alpha_{12} & 1 & 0 & \cdots & 0 \\
\left|\alpha_{2}\right|-1 & -\alpha_{21} & -\alpha_{22} & 0 & 1 & \cdots & 0 \\
\vdots & \vdots & \vdots & \vdots & \vdots & \ddots & \vdots \\
\left|\alpha_{m}\right|-1 & -\alpha_{m 1} & -\alpha_{m 2} & 0 & 0 & \cdots & 1
\end{array}\right)
$$


Lemma 8.3 Let $n=2$, and let $A$ and $B$ be as in (4) and (5). Then, the quadratic form $P_{A}(t)$ is given by the matrix $Q=\left(g\left(\alpha_{k}, \alpha_{j}\right)\right)_{k, j}$ where $1 \leq k, j \leq m$ and

$$
\begin{aligned}
g\left(\alpha_{k}, \alpha_{j}\right)= & \frac{1}{2}\left(\alpha_{k 1} \alpha_{j 2}\left(1-\alpha_{k 1}-\alpha_{j 2}\right)+\alpha_{k 2} \alpha_{j 1}\left(1-\alpha_{k 2}-\alpha_{j 1}\right)\right. \\
& \left.+\left(\alpha_{k 1} \alpha_{j 2}-\alpha_{k 2} \alpha_{j 1}\right)^{2}\right)
\end{aligned}
$$

Proof By Theorem 4.1, it suffices to consider the case $m=2$, which is a straightforward computation.

Let us introduce notation for the following $k \times k$-minor of the matrix $Q$ from Lemma 8.3:

$$
G_{k}\left(\begin{array}{c}
\alpha_{1}, \ldots, \alpha_{k} \\
\delta_{1}, \ldots, \delta_{k}
\end{array}\right)=\left|\begin{array}{ccc}
g\left(\alpha_{1}, \delta_{1}\right) & \cdots & g\left(\alpha_{1}, \delta_{k}\right) \\
\vdots & \ddots & \vdots \\
g\left(\alpha_{k}, \delta_{1}\right) & \cdots & g\left(\alpha_{k}, \delta_{k}\right)
\end{array}\right|
$$

From this point on, proving the above statements is a matter of endurance during computations. We avoid most details in this presentation. We invite the reader to verify the following claims (preferably using a computer).

Lemma 8.4 The polynomial $G_{k}$ vanishes identically if $k \geq 4$.

Proof The case $k>4$ follows from the case $k=4$ by a Laplace expansion. The case $k=4$ is a straightforward computation.

Thus, only the polynomials $G_{2}$ and $G_{3}$ are relevant for our investigation. The polynomial $G_{2}$ has, when expanded, 96 terms. The polynomial $G_{3}$ is simpler; it admits a factorization

$$
G_{3}\left(\begin{array}{c}
\alpha_{1}, \alpha_{2}, \alpha_{3} \\
\delta_{1}, \delta_{2}, \delta_{3}
\end{array}\right)=\frac{1}{4} H\left(\alpha_{1}, \alpha_{2}, \alpha_{3}\right) \cdot H\left(\delta_{1}, \delta_{2}, \delta_{3}\right)
$$

where $H\left(\alpha_{1}, \alpha_{2}, \alpha_{3}\right)$ is the following polynomial with (when expanded) 24 terms:

$$
\begin{aligned}
H\left(\alpha_{1}, \alpha_{2}, \alpha_{3}\right)= & \alpha_{11} \alpha_{12} \alpha_{22} \alpha_{31}\left(1-\alpha_{22}\right)\left(1-\alpha_{31}\right) \\
& -\alpha_{11} \alpha_{12} \alpha_{21} \alpha_{32}\left(1-\alpha_{21}\right)\left(1-\alpha_{32}\right) \\
& +\alpha_{12} \alpha_{21} \alpha_{31} \alpha_{32}\left(1-\alpha_{12}\right)\left(1-\alpha_{21}\right) \\
& -\alpha_{12} \alpha_{21} \alpha_{22} \alpha_{31}\left(1-\alpha_{12}\right)\left(1-\alpha_{31}\right) \\
& -\alpha_{11} \alpha_{22} \alpha_{31} \alpha_{32}\left(1-\alpha_{11}\right)\left(1-\alpha_{22}\right) \\
& +\alpha_{11} \alpha_{21} \alpha_{22} \alpha_{32}\left(1-\alpha_{11}\right)\left(1-\alpha_{32}\right) .
\end{aligned}
$$

Lemma 8.5 Assume that $0, e_{1}, e_{2}, \alpha_{1}$, and $\alpha_{2}$ are five distinct points in $\mathbb{R}^{2}$. Then, the polynomial $G_{2}$ vanishes for $\delta_{1}=\alpha_{1}$ and $\delta_{2}=\alpha_{2}$ if and only if there is a parabola containing the five points. 
Proof We assume that $\alpha_{11}\left(1-\alpha_{11}\right) \neq 0$, as this is the most difficult case. The cases $\alpha_{11}=0$ or $\alpha_{11}=1$ can be treated in analogous fashion. The general equation of a parabola passing through the points $0, e_{1}$, and $e_{2}$ is $P(x)=0$ where

$$
P(x)=a^{2} x_{1}^{2}+2 a b x_{1} x_{2}+b^{2} x_{2}^{2}-a^{2} x_{1}-b^{2} x_{2} .
$$

Requiring in addition that the parabola passes through $\alpha_{1}$ gives that, in projective coordinates

$$
[a: b]=\left[\alpha_{11} \alpha_{12} \pm \sqrt{\alpha_{11} \alpha_{12}\left(\alpha_{11}+\alpha_{12}-1\right)}: \alpha_{11}\left(1-\alpha_{11}\right)\right]
$$

(Recall that we assume $\alpha_{11}\left(1-\alpha_{11}\right) \neq 0$.) Let $P_{1}(x)$ and $P_{2}(x)$ denote the two parabolic equations obtained from the possible choices of signs. We leave it to the reader to verify that

$$
P_{1}\left(\alpha_{2}\right) P_{2}\left(\alpha_{2}\right)=4 \alpha_{11}^{2}\left(1-\alpha_{11}\right)^{2} G_{2}\left(\begin{array}{c}
\alpha_{1}, \alpha_{2} \\
\alpha_{1}, \alpha_{2}
\end{array}\right) .
$$

Remark 8.6 Assume that $A$, written in the form (4), is contained in a parabola $C$. For any indices $i, j$, and $k$, we obtain a quadratic polynomial

$$
R_{i j k}(x)=G_{2}\left(\begin{array}{c}
\alpha_{i}, \alpha_{j} \\
\alpha_{k}, x
\end{array}\right)
$$

Let us assume that $R_{121}$ is nontrivial, implying that the points $0, e_{1}, e_{2}, \alpha_{1}$, and $\alpha_{2}$ are in general position (in the sense that there is a unique conic passing through them). By Lemma 8.5, $R_{121}(x)$ vanishes for $x=\alpha_{2}$. It is straightforward to verify that $R_{121}(x)$ also vanishes for $x=0, e_{1}, e_{2}$, and $\alpha_{1}$. Hence, $R_{121}(x)$ defines the parabola $C$. In particular, $R_{121}(x)$ vanishes for all $\alpha \in A$.

Assume now that $A$ has at least six points, and in addition that $R_{123}(x)$ is nontrivial. We have that $R_{123}(x)$ vanishes at $x=\alpha_{2}$ and at $x=\alpha_{3}$, as it coincides (up to a constant) with $P_{232}\left(\alpha_{1}\right)$, respectively, $P_{323}\left(\alpha_{1}\right)$ for those values. It is straightforward to check that $R_{123}(x)$ also vanishes for $x=0, e_{1}$, and $e_{2}$. Hence, also $R_{123}(x)$ defines the parabola $C$. In particular, $R_{123}(x)$ vanishes for all $\alpha \in A$.

Lemma 8.7 Assume that $0, e_{1}, e_{2}, \alpha_{1}, \alpha_{2}$, and $\alpha_{3}$ are six distinct points in $\mathbb{R}^{2}$. Then, the polynomial $H\left(\alpha_{1}, \alpha_{2}, \alpha_{3}\right)$ from (6) vanishes if and only if there is a conic containing all six points.

Proof The general equation of a conic passing through the points $0, e_{1}$, and $e_{2}$ is $P(x)=0$ where

$$
P(x)=a x_{1}^{2}+b x_{1} x_{2}+c x_{2}^{2}-a x_{1}-c x_{2}
$$

Requiring in addition that the conic passes through $\alpha_{1}$ and $\alpha_{2}$ gives the coefficients, up to multiplication by a constant, 


$$
\left\{\begin{array}{l}
a=\alpha_{12} \alpha_{22}\left(\alpha_{21}-\alpha_{11}-\alpha_{12} \alpha_{21}+\alpha_{11} \alpha_{22}\right) \\
b=\alpha_{12} \alpha_{21}\left(1-\alpha_{12}\right)\left(1-\alpha_{21}\right)-\alpha_{11} \alpha_{22}\left(1-\alpha_{11}\right)\left(1-\alpha_{22}\right) \\
c=\alpha_{11} \alpha_{21}\left(\alpha_{12}-\alpha_{12} \alpha_{21}-\alpha_{22}+\alpha_{11} \alpha_{22}\right)
\end{array}\right.
$$

We leave it for the reader to verify that, with these coefficients, the polynomial $P(x)$ evaluated at $x=\alpha_{3}$ is equal to $H\left(\alpha_{1}, \alpha_{2}, \alpha_{3}\right)$.

Proof of Theorem 8.1 It is well known that for $n=2$ the point configuration $A$ is dual defective if and only if $A$ is a pyramid. This proves part (i) of the theorem.

If the codimension $m=1$, then $A$ consists of four points in the plane, through which two parabolas pass. It can be seen from (8) that the limiting case between real and nonreal parabolas is the case of a pyramid. Thus, this case follows from Example 3.3.

We now assume that $m>1$. Classical geometry says that there is a unique conic passing through five points in generic position (i.e., no four are colinear) in the plane. We allow the conic to be degenerate. Assuming that $A$ is not a pyramid, it has a subconfiguration $A_{1}$ of five points in generic position. Let $A_{1}$ constitute the first five columns of (4).

Let us first prove the relaxed statement, where we only consider the rank of the cuspidal form $P_{A}(t)$. It follows from Lemma 8.4 that the rank is at most three.

If the rank is at most two, then by Lemma 8.7 any choice of six points of $A$ is contained in a conic. However, the five points of $A_{1}$ determine a unique conic $C$. By adjoining the remaining points one by one, we conclude that $A$ is contained in the conic $C$. Conversely, if $A$ is contained in a conic, then $G_{3}$ vanishes for all $\alpha$ and $\delta$ by (6).

If the rank is at most one, then by Lemma 8.5 any choice of five points is contained in a parabola. In particular, the five points of $A_{1}$ are contained in a parabola $C$. Let $\alpha$ be an additional point. Then, by Lemma 8.7, $A_{1} \cup\{\alpha\}$ is contained in a conic. But $C$ is the unique conic containing $A_{1}$, so $\alpha \in C$. It follows that $A$ is contained in $C$. Conversely, if $A$ is contained in a parabola, then $G_{2}$ vanishes for all $\alpha$ and $\delta$ by Lemma 8.5 and Remark 8.6.

Let us now turn to the refined statement of Theorem 8.1 regarding signatures. For each class in the above list, it suffices to consider the minimal $m$ such that there is a point configuration in this class. Indeed, there is a "minimal" subconfiguration witnessing the class containing $A$, and by Theorem 4.1 we can delete the remaining points without altering the rank. In particular, parts (ii) and (iii) follow from the above discussion on codimension $m=1$.

Any two minimal configurations of one class can be continuously deformed to each other without leaving the class in question. Therefore, it suffices to consider one representative of each class. Since a generic configuration can be continuously deformed to a configuration contained either in an ellipse or in a parabola, part (vi) follows from parts (iv) and (v) and the fact that the rank is 3 in the generic case. Hence, we finish the proof with Examples 8.8 and 8.9.

Example 8.8 Consider the point configuration and Gale dual

$$
A=\left(\begin{array}{rrrrr}
1 & 1 & 1 & 1 & 1 \\
3 & -3 & 5 & 5 & -5 \\
0 & 0 & 4 & -4 & 4
\end{array}\right) \quad \text { and } \quad B^{\mathrm{T}}=\left(\begin{array}{rrrrr}
5 & -5 & -3 & 0 & 3 \\
-8 & 2 & 3 & 3 & 0
\end{array}\right)
$$


We obtain the cuspidal form $P_{A}(t)=-576\left(5 t_{1}-4 t_{2}\right)\left(5 t_{1}+4 t_{2}\right)$, which has signature $s_{A}=(1,1 ; 0)$.

Example 8.9 Consider the point configuration and Gale dual

$$
A=\left(\begin{array}{lllll}
1 & 1 & 1 & 1 & 1 \\
0 & 1 & 0 & 1 & 2 \\
0 & 0 & 1 & 2 & 1
\end{array}\right) \quad \text { and } \quad B^{\mathrm{T}}=\left(\begin{array}{lllll}
2 & -2 & -1 & 0 & 1 \\
2 & -1 & -2 & 1 & 0
\end{array}\right)
$$

We obtain the cuspidal form $P_{A}(t)=-4\left(t_{1}^{2}+t_{1} t_{2}+t_{2}^{2}\right)$, which has signature $s_{A}=$ $(0,2 ; 0)$.

Acknowledgements I would like to express my deep gratitude to Professor Alicia Dickenstein, Professor Ragni Piene, and Professor J. Maurice Rojas, for their helpful comments and encouragement. I also thank the anonymous referee who suggested several improvements to the manuscript.

Open Access This article is distributed under the terms of the Creative Commons Attribution 4.0 International License (http://creativecommons.org/licenses/by/4.0/), which permits unrestricted use, distribution, and reproduction in any medium, provided you give appropriate credit to the original author(s) and the source, provide a link to the Creative Commons license, and indicate if changes were made.

\section{References}

1. Bihan, F., Dickenstein, A.: Descartes' rule of signs for polynomial systems supported on circuits. arXiv:1601.05826 (2016)

2. Dickenstein, A., Feichtner, E.M., Sturmfels, B.: Tropical discriminants. J. Am. Math. Soc. 20(4), 1111-1133 (2007)

3. Dickenstein, A., Piene, R.: Higher order selfdual toric varieties. Ann. Mat. Pura Appl. 196(5), 17591777 (2017)

4. Dickenstein, A., Rojas, J.M., Rusek, K., Shih, J.: Extremal real algebraic geometry and $\mathscr{A}$ discriminants. Mosc. Math. J. 7(3), 425-452-574 (2007)

5. Esterov, A.: Newton polyhedra of discriminants of projections. Dicrete Comput. Geom. 44(1), 96-148 (2010)

6. Esterov, A.: Characteristic classes of affine varieties and Plücker formulas for affine morphisms. arXiv:1305.3234v7 (2013)

7. Forsgård, J., Nisse, M., Rojas, J.M.: New subexponential fewnomial hypersurface bounds. arXiv:1710.00481 (2017)

8. Gel'fand, I.M., Kapranov, M.M., Zelevinsky, A.V.: Discriminants, Resultants, and Multidimensional Determinants. Mathematics: Theory and Applications. Birkhäuser Boston Inc, Boston (1994)

9. Gomez, J., Niles, A., Rojas, J.M.: New complextiy bounds for certain fewnomial zero sets (extended abstract), Strobl, Austria (2007)

10. Kapranov, M.M.: A characterization of $A$-discriminantal hypersurfaces in terms of the logarithmic Gauss map. Math. Ann. 290(2), 277-285 (1991)

11. Katz, N.: Pinceaux de Lefschetz: théoreme d'éxistence. In: Lecture Notes in Mathematics, vol. 340, ch. XVII, pp. 212-253. Springer, Berlin (1973)

12. Khovanskiı̌, A.G.: Fewnomials. In: Translations of Mathematical Monographs, vol. 88. American Mathematical Society, Providence (1991) (Translated from the Russian by Smilka Zdravkovska)

13. Mikhalkin, E.N., Tsikh, A.: Singular strata of cuspidal type for the classical discriminant. Mat. Sb. 206(2), 119-148 (in Russian) (2015). (Translation in Sb. Math. 206(1-2), 282-310 (2015))

14. Müller, S., Feliu, E., Regensburger, G., Conradi, C., Shiu, A., Dickenstein, A.: Sign conditions for injectivity of generalized polynomial maps with applications to chemical reaction networks and real algebraic geometry. Found. Comput. Math 16(1), 69-97 (2016)

15. Rojas, J.M., Rusek, K.: A-Discriminants for complex exponents and counting real isotopy types. arXiv: 1612.03458 (2016) 